\title{
Effect of the Inhibition of Mitochondrial Creatine Kinase Activity on the Clinical Diagnosis of Suspected Acute Myocardial Infarction
}

\author{
Masato Maekawa*, ${ }^{1}$, Aya Sugiura ${ }^{1}$, Kunihiro Iwahara ${ }^{1}$, Yasuhiro Sakai ${ }^{2}$ and Kouji Kishi ${ }^{3}$ \\ ${ }^{1}$ Department of Laboratory Medicine, Hamamatsu University School of Medicine, Hamamatsu 431-3192, Japan \\ ${ }^{2}$ Elemental Technology Development Division, Sysmex Corporation, Kobe 651-2271, Japan \\ ${ }^{3}$ Product Development Division 2, Sysmex Corporation, Kobe 651-2271, Japan
}

\begin{abstract}
Background: Creatine kinase isoenzyme MB (CK-MB) activity using the immunoinhibition method has been measured in clinical laboratories. However, the presence of mitochondrial CK (MtCK) abnormally increases CK-MB activity when measured using the conventional method, which can lead to misdiagnosis of acute myocardial infarction (AMI). A new CK-MB activity assay kit that can inhibit MtCK activity was recently developed; here, we describe the performance of this kit.
\end{abstract}

Methods: The analytical performance of this new kit was investigated using 338 samples, and the correlation between results obtained using the new and conventional kits were analyzed.

Results: A good correlation $(r>0.993)$ was found between results obtained using the new and conventional kits in the sera from patients with obvious AMI $(n=91)$. However, a poor correlation exists between results obtained using the new and conventional kits in samples from patients without AMI. Electrophoretic CK isoenzyme analysis confirmed that the appearance of MtCK in nearly all samples from patients with malignant tumors makes poor correlation.

Conclusion: Inhibiting MtCK activity is important for diagnosing AMI. The new method may provide a more accurate value of CK-MB activity for clinical use.

Keywords: Biological markers, diagnostic reagent kits, creatine kinase MB, mitochondrial creatine kinase, myocardial infarction.

\section{INTRODUCTION}

Currently, the diagnosis of acute myocardial infarction (AMI) is based on changes in electrocardiograms and elevated levels of biochemical markers, particularly creatine kinase isoenzyme MB (CK-MB) $[1,2]$. CK-MB activity is an early marker of AMI, and damaged myocardium shows substantial elevations in the levels of this marker. Although cardiac troponin levels, which show greater sensitivity and selectivity than CK-MB activity levels in detecting minor myocardial damage [3], have been recommended to be the new laboratory standards for AMI diagnosis, CK-MB activity measurements are still considered useful in detecting myocardial infarction.

While CK-MB levels are considered as one of the early diagnostic markers of AMI, CK-MB activity assays performed using the immunoinhibition method remain useful in clinical laboratories. However, the presence of mitochondrial CK (MtCK), CK-BB, or CK-immunoglobulin complex (macro CK type 1) in patient serum can result in abnormally high CK-MB values in the immunoinhibition assay and lead to misdiagnosis of AMI [4-8]. This is because the anti-

*Address correspondence to this author at the 1-20-1 Handayama, Higashiku, Hamamatsu 431-3192, Japan; Tel: +81-53-435-2721;

Fax: +81-53-435-2096; E-mail: mmaekawa@ hama-med.ac.jp
CK-M antibody included in the CK-MB activity reagents can inhibit the CK-M subunit but not MtCK or macro CK type 1. Lee $e t$ al reported that the frequency of appearance of MtCK in serum was considerably higher than that of the macro CK type 1 [7]. Therefore, to ensure accurate determination of CK-MB activity, it is important to inhibit the activity of $\mathrm{MtCK}$.

The L-System CK-MB MtO (Sysmex Corporation, Kobe, Japan), a new reagent kit that can be used to measure CKMB activity and inhibit MtCK activity, was developed recently $[9,10]$. Here, we evaluated the clinical usefulness of this new CK-MB reagent, which includes anti-MtCK antibodies and abrogates the effect of MtCK activity in the serum, and confirmed the clinical efficacy of inhibition of MtCK activity.

\section{MATERIALS AND METHODOLOGY}

\section{Samples}

A correlation study was conducted using 338 samples of residual sera from patients after completing the requested laboratory tests at the Hamamatsu University School of Medicine, University Hospital (Hamamatsu, Japan). Residual samples contained sera from patients with obvious AMI $(n=91)$ and without AMI, including patients with cancer, 
hepatitis, and cardiac disease other than AMI $(n=247)$. Samples were anonymized and stored at $-80^{\circ} \mathrm{C}$ until the assay. Procedures used in this study were conducted in accordance with the Declaration of Helsinki of 1975 and the amendments of 1983 .

\section{Materials}

CK-MB and CK activities were measured using the LSystem CK-MB MtO, conventional CK, and CK-MB activity assay kits. We measured CK-MB activity using CK-MB Liquid (Roche Diagnostics, Tokyo, Japan) and measured CK activity using a commercially available CK kit (CK-NAC Liquid; Roche Diagnostics).

L-System CK-MB MtO is a novel CK-MB assay kit; this kit includes an anti-CK-M polyclonal antibody (goat) and 2 types of anti-MtCK monoclonal antibody (mouse) against ubiquitous MtCK (uMtCK) and sarcomeric MtCK (sMtCK) in a reagent set for determining CK activity [10]. Anti-CK-M antibody and 2 types of anti-MtCK antibody can inhibit approximately $3000 \mathrm{U} / \mathrm{L}$ CK-MM and $500 \mathrm{U} / \mathrm{L}$ MtCK, respectively. The kit for determining CK-MB activity consists of 2 types of reagents.

\section{Principle of L-System CK-MB MtO}

Sera samples were incubated with reagents containing antibodies against CK-M subunit and MtCKs. The CK-M subunit and MtCKs in samples were inhibited by Anti-CK-M antibody and anti-MtCK antibodys. Creatine phosphate was added to initiate the reaction, and residual CK-B subunit activity was measured.

\section{Instruments}

CK-MB and CK activities were measured using a Hitachi 7700(P) automated biochemical analyzer (Hitachi HighTechnologies, Tokyo, Japan). Assays for CK-MB activity and CK activity were performed in accordance with the manufacturer's protocols.

\section{CK-MB Assay}

First, $12.9 \mu \mathrm{L}$ of sample was incubated with $180 \mu \mathrm{L}$ of reagent 1 for $2.5 \mathrm{~min}$ at $37^{\circ} \mathrm{C}$ and the absorbance change per minute of blank sample was measured at $340 / 700 \mathrm{~nm}$ $\left(\Delta \mathrm{A}_{\text {blank }}\right)$ using an H-7700 automated biochemical analyzer. The reaction was initiated by addition of $45 \mu \mathrm{L}$ of reagent 2 . After $2.5 \mathrm{~min}$, the absorbance change per minute of sample at $340 / 700 \mathrm{~nm}\left(\Delta \mathrm{A}_{\text {sample }}\right)$ was measured. The absorbance change per minute was calculated by subtraction of $\Delta \mathrm{A}_{\text {blank }}$ from $\Delta \mathrm{A}_{\text {sample }}$. CK-MB activity was determined using a calibration curve constructed using CK-MB calibrator, Calibzyme CK (MB) (Sysmex Corporation), which contained a CK-MB curve derived from human cardiac muscle.

\section{Analysis of CK Isoenzyme Using Electrophoresis}

Serum CK isoenzymes were separated electrophoretically using a cellulose acetate membrane (Titan III-Lipo; Helena, Saitama, Japan). HR Buffer (Helena) was used as the migration buffer. Electrophoresis was carried out at $190 \mathrm{~V}$ for 30 min under cold conditions. Next, the membrane was stained with a CK isoenzyme reagent (Titan CK-S; Helena). Staining procedures for $\mathrm{CK}$ isoenzymes were performed in accordance with the manufacturer's protocol. A control marker containing CK-MM, CK-MB, and CK-BB was used for each run.

\section{Statistical Analyses}

Statistical analyses were performed using Microsoft Excel 2003 (Microsoft, Redmond, WA, USA).

\section{RESULTS}

\section{Error Calculations}

Within-run $(n=20)$ and between-day errors (15 days, $n=$ 1) were estimated by performing measurements on 1 commercial control serum sample and 4 pooled serum samples (Table 1). Calibration was performed on each test day. The within-run errors (CV) determined using 1 commercial control serum sample and 2 pooled serum samples with different

Table 1. Within-run ( $n=20$ ) and between-day Errors (15 Days) of L-system CK-MB MtO

A)

Within-run Imprecision $(n=20)$

\begin{tabular}{|c|c|c|c|}
\hline & Control Serum & Pooled Serum 1 & Pooled Serum 2 \\
\hline \hline Mean (U/L) & 56.6 & 31.9 & 132.0 \\
\hline SD (U/L) & 0.71 & 1.12 & 1.66 \\
\hline CV (\%) & 1.26 & 3.50 & 1.26 \\
\hline
\end{tabular}

B) Between-day Imprecision (15 Days)

\begin{tabular}{|c|c|c|c|}
\hline & Control Serum & Pooled Serum 3 & Pooled Serum 4 \\
\hline \hline Mean (U/L) & 58.0 & 30.7 & 126.4 \\
\hline SD $(\mathrm{U} / \mathrm{L})$ & 1.21 & 1.42 & 1.99 \\
\hline $\mathrm{CV}(\%)$ & 2.09 & 4.64 & 1.57 \\
\hline
\end{tabular}

Within-run $(n=20)$ and between-day errors (15 days, $n=1)$ were determined using 1 commercial control serum sample (CK isozyme control [Sysmex Corporation]) and 2 pooled serum samples. Calibration was performed on each test day. 
CK-MB activities were 1.26\% (CK-MB 57 U/L), 3.5\% (CKMB $32 \mathrm{U} / \mathrm{L}$ ), and $1.26 \%$ (CK-MB $132 \mathrm{U} / \mathrm{L}$ ), respectively. The between-day errors (CV) were $2.1 \%$ (CK-MB $58 \mathrm{U} / \mathrm{L}$ ), 4.6\% (CK-MB $31 \mathrm{U} / \mathrm{L}$ ), and 1.57\% (CK-MB $126 \mathrm{U} / \mathrm{L}$ ), respectively.

\section{Linearity Studies}

Linearity of the L-System CK-MB MtO was evaluated by preparing sequential dilutions of CK-MB high-activity samples (Scripps Laboratories, San Diego, USA) and a human serum pool. A good linear response up to $2000 \mathrm{U} / \mathrm{L}$ was obtained. This response was fitted to the following linear regression equations $(95 \%$ confidence intervals in parentheses): $\mathrm{y}=[0.997$ (0.99676 to 0.99687$) \mathrm{x}+4.8$ (0.7 to 9.0$)]$ $\mathrm{U} / \mathrm{L}, \mathrm{r}=1.000, \mathrm{Sy} \mid \mathrm{x}=3.26 \mathrm{U} / \mathrm{L}$ for low CK-MB activity (maximum CK-MB activity, $345 \mathrm{U} / \mathrm{L}$ ) and $\mathrm{y}=[1.023$ $(1.02346$ to 1.02348$) \mathrm{x}-5.0(-21.8$ to 11.9$)] \mathrm{U} / \mathrm{L}, \mathrm{r}=0.999$, Sy|x $=9.64 \mathrm{U} / \mathrm{L}$ for high CK-MB activity (approximately $2046 \mathrm{U} / \mathrm{L})$.

\section{Interfering Substances}

We evaluated the interference of unconjugated bilirubin, conjugated bilirubin, and hemolysis using this method. Bilirubin dissolved with $0.1 \mathrm{~mol} / \mathrm{L} \mathrm{NaOH}$ and hemolysis dissolved with deionized water were added to pooled serum (1:9, by volume). No interference of unconjugated bilirubin, conjugated bilirubin, or hemolysis was observed up to 19 $\mathrm{mg} / \mathrm{dL}, 21 \mathrm{mg} / \mathrm{dL}$, and $484 \mathrm{mg} / \mathrm{dL}$, respectively.

\section{Comparison between Conventional and L-system CK- MB MtO Kits in Sera from Patients with Obvious AMI and Without AMI}

Fig. (1) shows the correlation between results obtained using the conventional assay reagent and new reagent by using 91 serum samples from patients with obvious AMI. Good agreement was observed between the new CK-MB activity assay kit and conventional assay kits $(\mathrm{y}=[0.958 \mathrm{x}-$ 9.2] U/L; $r=0.996 ;$ Sy|x $=8.29 \mathrm{U} / \mathrm{L}$ ). However, we observed a poor correlation between the results obtained using new and conventional kits in samples containing sera from patients without AMI $(n=247)$ (Fig. 2). Although values from some samples were concordant in CK-MB activity between the new and conventional kits, others were discordant. Some CK-MB values assayed by the conventional method were above $1000 \mathrm{U} / \mathrm{L}$, whereas those determined using the new kit were less than $50 \mathrm{U} / \mathrm{L}$. The new CK-MB kit can inhibit MtCK activity using an anti-MtCK antibody that is included in the kit, but the conventional kit cannot inhibit MtCK because it does not contain this antibody. Variation in CK-MB values was attributed to the presence of MtCK activity in sera from patients without AMI. These discordant samples included 16 samples from patients with malignancy, 2 from patients with hepatic damage, and 4 from patients with heart muscle damage (heart failure, cardiomyopathy, or congenital cardiac anomaly).

Table 2 shows CK and CK-MB activities in the 22 discordant samples described above, determined using the new and conventional kits. Calculated MtCK activities are also shown. In most samples from patients with malignancy as-

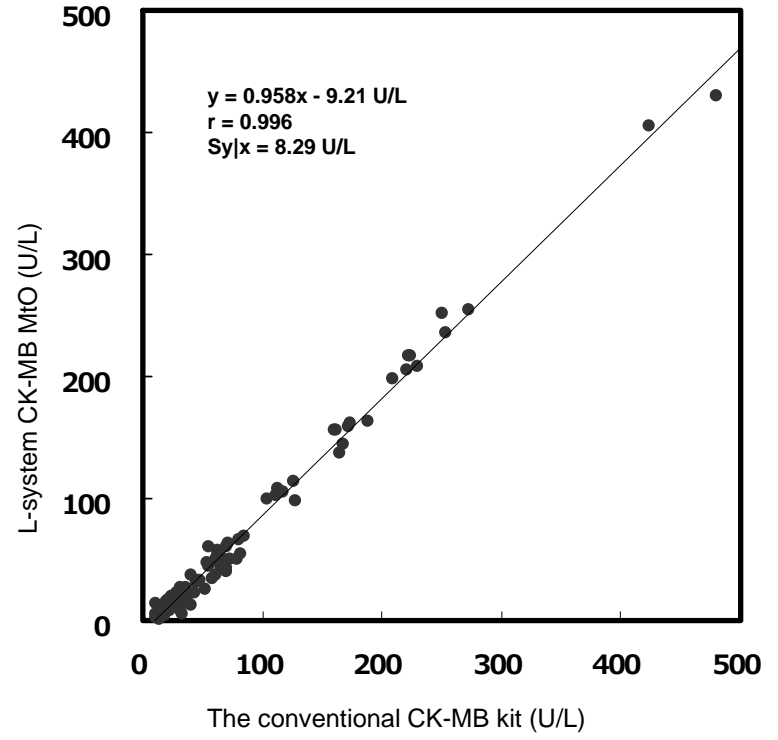

Fig. (1). Correlation studies in 91 serum samples from patients with obvious AMI.

In correlation studies, we used 91 samples of residual sera from patients with obvious AMI after completing the requested laboratory test. A good correlation was observed between the new and conventional kits.

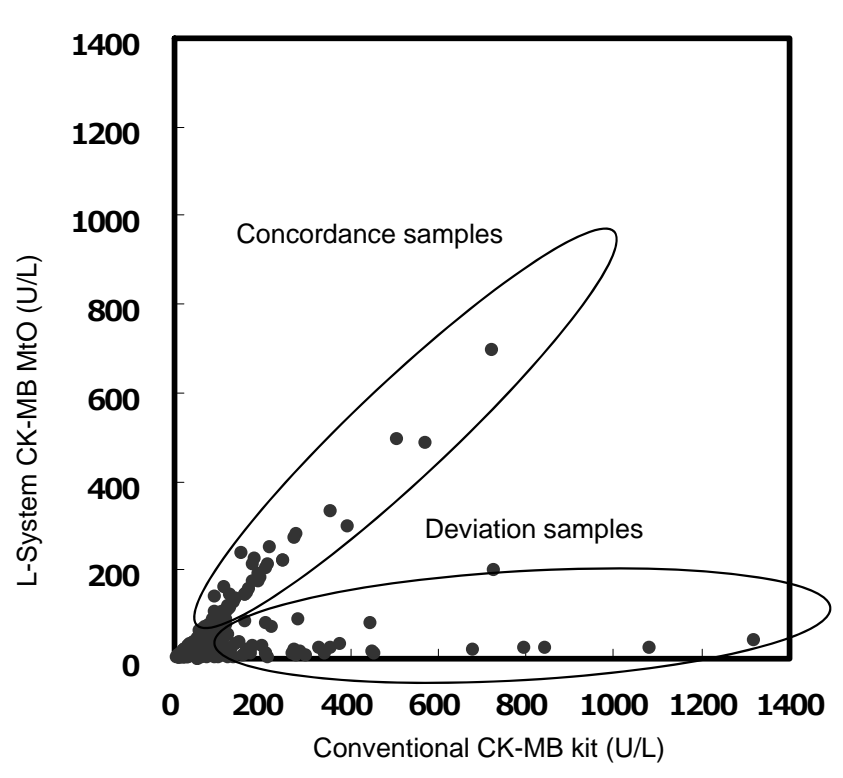

Fig. (2). Correlation studies in samples from patients without AMI. In correlation studies, 247 samples of residual sera from patients without AMI after they completed the requested laboratory test were used. Although some samples were concordant between the new and conventional kits, others were discordant. This discordance was attributed to MtCK activity in the sera of patients without AMI.

sayed using the conventional kit, the $\mathrm{CK}-\mathrm{MB} / \mathrm{CK}$ ratio was greater than $100 \%$. However, there were no samples in 
Table 2. Clinical Disease Associated with CK and CK-MB Activities in Discordant Samples

\begin{tabular}{|c|c|c|c|c|c|c|c|c|}
\hline \multirow[b]{2}{*}{ No } & \multirow[b]{2}{*}{ Category } & \multirow{2}{*}{$\begin{array}{c}\text { CK } \\
\text { Activity } \\
\text { (U/L) }\end{array}$} & \multicolumn{2}{|c|}{ The Conventional Method } & \multicolumn{2}{|c|}{ L-System CK-MB MtO } & \multirow{2}{*}{$\begin{array}{c}\text { MtCK } \\
\text { Activity } \\
\text { (U/L) }\end{array}$} & \multirow[b]{2}{*}{ Clinical Disease } \\
\hline & & & $\begin{array}{c}\text { CK-MB } \\
\text { activity } \\
\text { (U/L) }\end{array}$ & $\begin{array}{c}\text { CK- } \\
\text { MB/CK } \\
(\%)\end{array}$ & $\begin{array}{c}\text { CK-MB } \\
\text { activity } \\
(\mathbf{U} / \mathbf{L})\end{array}$ & $\begin{array}{c}\text { CK- } \\
\text { MB/CK } \\
(\%)\end{array}$ & & \\
\hline 1 & \multirow{12}{*}{ Cancer } & 226 & 49.2 & 21.8 & 11.0 & 4.9 & 15.3 & Colorectal cancer \\
\hline 2 & & 55 & 121.5 & 220.9 & 18.1 & 32.9 & 46.9 & Colorectal cancer \\
\hline 4 & & 198 & 285.9 & 144.4 & 18.8 & 9.5 & 117.0 & Colon cancer \\
\hline 5 & & 151 & 119.4 & 79.1 & 55.0 & 36.4 & 25.6 & Endometrial cancer, Ovarian cancer \\
\hline 6 & & 109 & 59.5 & 54.6 & 2.3 & 2.1 & 25.6 & Pancreatic cancer \\
\hline 10 & & 542 & 1318.6 & 243.3 & 44.3 & 8.2 & 560.3 & Gastric cancer \\
\hline 11 & & 144 & 207.4 & 144.0 & 14.8 & 10.3 & 85.0 & Gastric cancer \\
\hline 12 & & 340 & 794.3 & 233.6 & 27.6 & 8.1 & 346.5 & Gastric cancer \\
\hline 13 & & 64 & 122.7 & 191.7 & 14.7 & 23.0 & 43.5 & Rectal cancer \\
\hline 14 & & 136 & 328.1 & 241.3 & 27.5 & 20.2 & 133.6 & Rectal cancer \\
\hline 15 & & 73 & 156.8 & 214.8 & 10.7 & 14.7 & 64.3 & Breast cancer \\
\hline 16 & & 791 & 83.9 & 10.6 & 12.7 & 1.6 & 31.5 & Hepatoma \\
\hline 21 & \multirow{2}{*}{$\begin{array}{l}\text { Cardiac } \\
\text { disease }\end{array}$} & 146 & 271.9 & 186.2 & 23.4 & 16.0 & 111.3 & $\begin{array}{c}\text { Cardiac failure, Dilated cardiomy- } \\
\text { opathy }\end{array}$ \\
\hline 22 & & 207 & 61.8 & 29.9 & 6.7 & 3.2 & 24.4 & Congenital cardiac anomaly \\
\hline
\end{tabular}

which CK-MB activity was greater than total CK activity, as determined using the new kit.

\section{CK Isoenzyme Electrophoresis of the Discordant Sam- ples in the Correlation Study}

CK isoenzyme electrophoresis using a discordant sample was performed to identify the presence of MtCK (Fig. 3). CK-MB activity of the sample in this figure was nearly undetectable by electrophoresis (lane 2). CK-MM and MtCK activities were detected by electrophoresis (lane 2). The MtCK band disappeared after treatment with anti-uMtCK antibody (lane 3) but not after treatment with anti-sMtCK antibody (lane 4). However, the CK-MM band could not be detected after treatment with anti CK-M antibody (lane 6). Disappearance of the electrophoretic band indicated that the CK isoenzyme was inhibited by the antibody. Therefore, the electropherogram in Fig. 3 shows that CK-MB was not present, but both uMtCK and CK-MM were present in this sample.

Fig. (4) shows the CK isoenzyme electrophoresis pattern of samples that were concordant between the new kit and conventional kits. These samples contained macro CK type 1 and CK-BB. Good correlation was observed between the results obtained using new and conventional kits, even if macro CK type 1 or CK-BB was present in the serum.

\section{DISCUSSION AND CONCLUSION}

MtCK was first discovered by Jacobus and Lehninger [11]; it is localized in the outer mitochondrial membrane, effectively facilitating the conversion of mitochondrial ATP to cytosolic phosphocreatine $[12,13]$. There are $2 \mathrm{MtCK}$ 


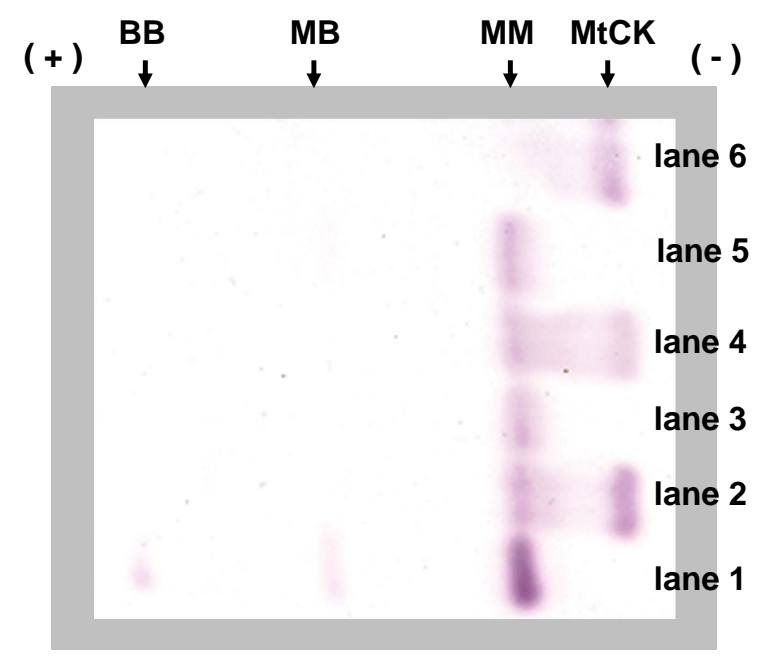

Fig. (3). CK isoenzyme electrophoresis of the discordant sample in the correlation study.

Lane 1, CK isoenzyme controls (CK-MM, CK-MB, and CK-BB); lane 2, non-treated sample; lane 3, sample treated with anti-uMtCK antibody; lane 4, sample treated with anti-sMtCK antibody; lane 5, sample treated with anti-uMtCK antibody and anti-sMtCK antibody; lane 6, sample treated with anti-CK-M antibody.

Activity staining for $\mathrm{CK}$ was performed after electrophoresis on cellulose acetate membrane using a sample that was discordant between the new and conventional kits. CK and CK-MB activities assayed using the new kit and conventional kits in this sample were $450 \mathrm{U} / \mathrm{L}, 27.5 \mathrm{U} / \mathrm{L}$, and 1082.1 U/L, respectively. Disappearance of the MtCK band indicates that uMtCK was inhibited by anti-uMtCK antibody and that uMtCK was present in this sample (lane 3). sMtCK was not present in this sample because the MtCK band was detected after treatment with anti-sMtCK antibody (lane 4). The band in lane 2 indicates that CK-MB was not present in this sample, but CK-MM was present in this sample (lane 6). Therefore, CK$\mathrm{MM}$ and uMtCK were present in this sample, and CK-MB and sMtCK was not present.

isoenzymes: (1) sMtCK, which exists in the striated muscle and (2) uMtCK, which is present in most tissues or cells such as the kidney, brain, and spermatozoa [14]. Lee et al. reported that the frequency of MtCK appearance in the serum was considerably higher than that of the macro CK type 1 [7]. Therefore, it is important to remove MtCK activity causing false positive findings if $\mathrm{CK}-\mathrm{MB}$ is to be performed more specifically.

There were differences in CK-MB values in some samples assayed by the new and conventional kits, although other values from samples assayed using the new kit were concordant with those determined using the conventional kits. CK-MB activity in the discordant samples was no more than $50 \mathrm{U} / \mathrm{L}$ using the new assay kit, which can inhibit MtCK, but this value was higher than $100 \mathrm{U} / \mathrm{L}$ using the conventional assay kit, which cannot inhibit MtCK. The MtCK band in the CK isoenzyme electrophoretic analysis disappeared after treatment with anti-MtCK antibody. Thus, MtCK appeared to be present in these samples. A good correlation between the results obtained using the new kit and the conventional kit was observed using sera from patients

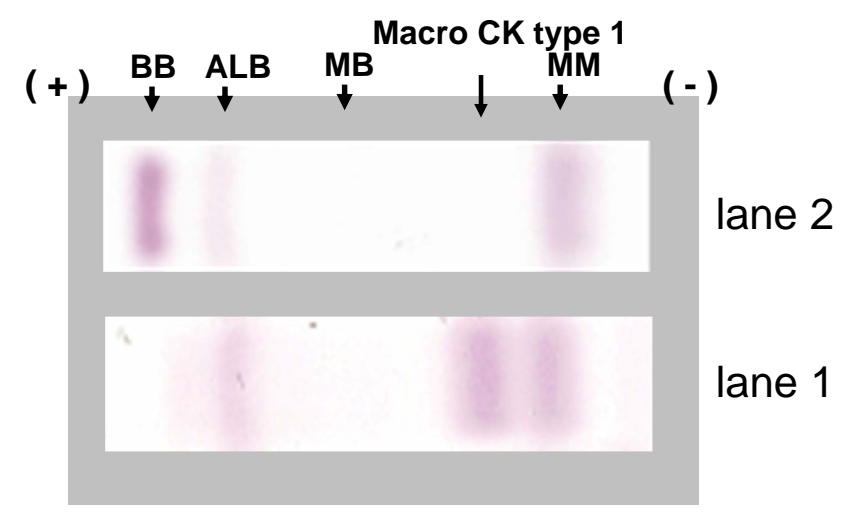

Fig. (4). CK isoenzyme electrophoresis of concordant samples in the correlation study.

$\mathrm{CK}$ isoenzyme electrophoresis of the concordant samples in the correlation study was performed. Activity staining of CK was performed after electrophoresis on a cellulose acetate membrane. Macro CK type 1 was present in the sample shown in lane 1 and CK-BB was present in the sample shown in lane 2. CK-MB was not present in these samples. Albumin bands (ALB) were observed by the staining because much more amounts of the sample than those in Fig. 3 were applied on the membrane.

with obvious AMI. The intercept of the linear regression equation was approximately $9 \mathrm{U} / \mathrm{L}$ and appeared to correspond to MtCK activity in patient sera. MtCK activity is present in the serum of healthy individuals, and it was 3.8 $\mathrm{U} / \mathrm{L}$ in an immunoinhibition assay [10]. MtCK activity in serum was calculated by subtracting CK-B activity determined using the new method from that determined using the conventional kit. This difference was divided by 2 because CK-MB activity was measured by doubling residual CK-B activity. Therefore, the value of $3.8 \mathrm{U} / \mathrm{L}$ for MtCK activity derived from an immunoinhibition assay corresponded to a $\mathrm{CK}-\mathrm{MB}$ activity of 7.6 U/L and was nearly the same as the intercept of the linear regression equation. Inhibiting $\mathrm{MtCK}$ activity improved the selectivity of the CK-MB activity assay.

In contrast, $\mathrm{CK}-\mathrm{MB}$ activity in some samples that were concordant between the new and conventional kits was higher than $50 \mathrm{U} / \mathrm{L}$, but undetectable by electrophoresis $(0$ $\mathrm{U} / \mathrm{L})$. These samples contained macro $\mathrm{CK}$ type 1 or CK-BB. CK-MB activity assayed using the new kit corresponded to the sum of CK-MB, macro CK type 1, and CK-BB. The new kit incorrectly measured CK-MB activity in samples in which CK-BB or macro CK type 1 was present.

Of all human tissues examined, the cardiac muscle contained the highest CK-MB activity, and approximately $22 \%$ of total CK activity was accounted for by CK-MB activity [15]. Therefore, CK-MB activity in the serum appears to be less than $22 \%$ of the total CK activity, even if CK-MB activity in the serum increased owing to the presence of AMI. Thus, samples for which the CK-MB/CK ratio was more than $22 \%$ may have contained other CK isoenzymes (MtCK, macro $\mathrm{CK}$ type 1 , or $\mathrm{CK}-\mathrm{BB}$ ). The $\mathrm{CK}-\mathrm{MB} / \mathrm{CK}$ ratio in sera from most patients with malignancy, hepatic damage, or heart muscle damage was more than $22 \%$, as determined using the conventional method. The $\mathrm{CK}-\mathrm{MB} / \mathrm{CK}$ ratio of 
samples (No. 2, 3, 5; Table 2) was more than $22 \%$ using the new kit. However, some samples for which the CK-MB/CK ratio was below $22 \%$ contained macro $\mathrm{CK}$ type 1 or $\mathrm{CK}-\mathrm{BB}$, as determined using the new CK-MB reagent. Therefore, it may be necessary to determine a correct cutoff for the CK$\mathrm{MB} / \mathrm{CK}$ ratio to determine whether macro $\mathrm{CK}$ type 1 or $\mathrm{CK}$ $\mathrm{BB}$ is present in some samples and gives the potential of a false-positive diagnosis using the new CK-MB reagent.

In conclusion, inhibiting MtCK activity occurring in specimen material is important in the clinical diagnosis of AMI. A new CK-MB kit, which can inhibit MtCK, can be used to estimate CK-MB activity more accurately than conventional kits because conventional kits do not inhibit MtCK and exhibit a high rate of false positivity for CK-MB activity. A diagnostic reagent that includes anti-MtCK antibody can provide clinicians with a more accurate value of $\mathrm{CK}-\mathrm{MB}$ activity. Thus, the new kit described here will improve the clinical diagnosis of AMI compared with the conventional method.

\section{ACKNOWLEDGEMENT}

None Declared.

\section{CONFLICT OF INTEREST}

None Declared.

\section{REFERENCES}

[1] Wu, A.H.B.; Schwartz, J.G. Update on creatine kinase isoenzyme assays. Diagn. Clin. Test, 1989, 27, 16-20.

[2] Zaninotto, M.; Altinier, S.; Lachin, M.; Carraro, P.; Plebani, M. Fluoroenzymometric method to measure cardiac troponin I in sera of patients with myodicardial infarction. Clin. Chem., 1996, 42, 1460-1466.

[3] Katus, H.A.; Looser, S.; Hallermayer, K.; Remppis, A.; Scheffold, T.; Borgya, A.; Essig, U.; Geuß, U. Development and in vitro characterization of a new immunoassay of cardiac Troponin T. Clin. Chem., 1992, 38, 386-393.
[4] Lederer, W.H.; Gerstbrein, H.L. Creatine kinase isoenzyme BB activity in serum of a patient with gastric cancer. Clin. Chem., 1976, 22, 1748-1749.

[5] Feld, R.D.; Witte, D.L. Presence of creatine kinase BB isoenzyme in some patients with prostatic carcinoma. Clin. Chem., 1977, 23, 1930- 1932.

[6] Pudek, M.R.; Jacobson, B.E. Falsely negative laboratory diagnosis for myocardial infarction owing to the concurrent presence of macro creatine kinase and macro lactate dehydrogenase. Clin. Chem., 1982, 28, 2434- 2437.

[7] Lee, K.N.; Csako, G.; Bernhardt, P.; Elin, R.J. Relevance of macro creatine kinase type 1 and type 2 isoenzymes to laboratory and clinical data. Clin. Chem., 1994, 40, 1278-1283.

[8] Schmid, H.; Mühlbayer, D.; Röling, J.; Sternfeld, T.; Jülg, B.; Schlattner, U.; Nelson, P.J.; Bogner, J.R.; Wallimann, T.; Goebel, F. Macroenzyme creatine kinase (CK) type 2 in HIV-infected patients is significantly associated with TDF and consists of ubiquitous mitochondrial CK. Antivir. Ther., 2006, 11, 1071-1080.

[9] Sakai, Y.; Yamashita, K.; Kajita, T.; Kishi, K.; Hoshino, T. Development and clinical application of NEW CK-MB reagent using anti-mitochondrial creatine kinase (MtCK) antibody [AACC 2005 Annual Meeting Abstracts]. Clin. Chem., 2005, 51, A-49.

[10] Hoshino, T.; Sakai, Y.; Yamashita, K.; Shirahase, Y.; Sakaguchi, K.; Asaeda, A.; Kishi, K.; Schlattner, U.; Wallimann, T.; Yanai, M.; Kumasaka, K. Development and performance of an enzyme immunoassay to detect creatine kinase isoenzyme MB activity using anti-mitochondrial creatine kinase monoclonal antibodies. Scand. J. Clin. Lab Invest., 2009, 69, 687-695.

[11] Jacobus, W.E.; Lehninger, A.L. Creatine kinase of rat heart mitochondria. J. Biol. Chem., 1973, 248, 4803- 4810.

[12] Qin, W.; Khuchua, Z.; Boero, J.; Payne, R.M.; Strauss, A.W. Oxidative myocytes of heart and skeletal muscle express abundant sarcomeric mitochondrial creatine kinase. Histochem. J., 1999, 31, 357-365.

[13] Saks, V.A.; Kupriyanov, V.V.; Elizarova, G.V.; Jacobus, W.E. Studies of energy transport in heart cells: the importance of creatine kinase localization for the coupling of mitochondrial phosphorylcreatine production to oxidative phosphorylation. J. Biol. Chem. 1980, 255, 755-763.

[14] Haas, R.C.; Strauss, A.W. Separate nuclear genes encode sarcomere-specific and ubiquitous human mitochondrial creatine kinase isoenzyme. J. Biol. Chem., 1990, 265, 6921-6927.

[15] Tsung, S.H. Creatine kinase isoenzyme patterns in human tissue obtained at surgery. Clin. Chem., 1976, 22, 173-175.

(C) Maekawa et al.; Licensee Bentham Open.

This is an open access article licensed under the terms of the Creative Commons Attribution Non-Commercial License (http://creativecommons.org/licenses/ by-nc/3.0/) which permits unrestricted, non-commercial use, distribution and reproduction in any medium, provided the work is properly cited. 